\title{
Flax (Linum usitatissimum L.) response to non-optimal acidity and zinc deficiency
}

\author{
A. Dmitriev ${ }^{1 *}$, G. Krasnov ${ }^{1}$, A. Zyablitsin ${ }^{1}$, T. Rozhmina ${ }^{1,2}$, R. Novakovskiy ${ }^{1}$, \\ P. Kezimana ${ }^{1}$, A. Kudryavtseva ${ }^{1}$, N. Melnikova ${ }^{1}$ \\ ${ }^{1}$ Engelhardt Institute of Molecular Biology RAS, Moscow, Russia \\ ${ }^{2}$ All-Russian Research Institute for Flax, Torzhok, Russia \\ *e-mail:Alex_245@mail.ru
}

Key words: flax, Linum usitatissimum, zinc deficiency, stress response, high-throughput sequencing

\begin{abstract}
Motivation and Aim: Flax (Linum usitatissimum L.) is grown for fiber and seed production. Unfavorable environments, such as nutrient deficiency and non-optimal soil acidity, decrease quantity and quality of yield. Cultivation of resistant to stress varieties can significantly reduce the crop losses. Understanding the mechanisms of flax response to the stresses and identification of resistance gene candidates will help in breeding of improved cultivars. In the present work, response of flax plants to increased $\mathrm{pH}$ level and
\end{abstract} zinc (Zn) deficiency was studied.

Methods and Algorithms: About 200 plants of flax cultivars, Norlin (resistant) and Mogilevsky (sensitive), were grown under control conditions, Zn deficiency (1000-fold reduced $\mathrm{Zn}$ content), increased $\mathrm{pH}$ level (7.5), and both $\mathrm{Zn}$ deficiency and $\mathrm{pH}$ 7.5. Total RNA was isolated from root tips. Illumina TruSeq Stranded Total RNA Sample Prep Kit was used for library preparation. The cDNA libraries were sequenced on Illumina NextSeq500 with 80-nucleotide read length. Transcriptome assembly, annotation, and expression analysis was performed using Trinity, Trinotate, TransDecoder, Bowtie2, and RSEM as described earlier [1].

Results: High-throughput sequencing of 16 cDNA libraries of flax cultivars, grown under control conditions, increased $\mathrm{pH}$ level, Zn deficiency, and both stresses simultaneously, was performed, and about 40 million reads were obtained for each experiment type. At $\mathrm{pH} 7.5$, upregulation of stress-related genes and downregulation of genes, associated with the biogenesis of the cell wall, were revealed in flax plants. Under $\mathrm{Zn}$ deficiency, expression alterations were identified for genes involved in transmembrane transport and photosynthesis. Besides, distinct expression changes were revealed for flax genotypes with diverse resistance to studied stresses.

Conclusion: We identified genes with expression alterations in flax under non-optimal acidity and Zn deficiency. These genes are involved in diverse processes, including cell wall biogenesis, transmembrane transport, and photosynthesis, and could play an important role in flax response to the studied stresses. Moreover, genes with distinct expression changes between resistant and sensitive genotypes could determine the mechanisms of flax resistance to non-optimal acidity and $\mathrm{Zn}$ deficiency.

Acknowledgements: This work was financially supported by the Russian Science Foundation, grant 16-16-00114.

\section{References}

1. Dmitriev A.A. et al. (2016) Glutathione S-transferases and UDP-glycosyltransferases are involved in response to aluminum stress in flax. Front. Plant Sci. 7:1920. DOI 10.3389/fpls.2016.01920. 